\title{
Prototipo electrónico simple y de bajo costo para rastreo de vehículos basado en GPS
}

\section{Simple and low-cost electronic prototype for GPS-based vehicle tracking}

\author{
MORALES-HERNÁNDEZ, Maricela†*, CABALLERO-JULIAN, Franco Gabriel, WONG-DÍAZ, \\ Antonio Erick y AGUILAR-ORTIZ, Gabriela
}

Instituto Tecnológico de Oaxaca / Tecnológico Nacional de México

ID $1^{\mathrm{er}}$ Autor: Maricela, Morales-Hernández / ORC ID: 0000-0002-3521-2041, CVU CONACYT ID: 731036

ID $1^{\text {er }}$ Coautor: Franco Gabriel, Caballero-Julian / ORC ID: 0000-0002-5924-7759, CVU CONACYT ID: 88993

ID $2^{\text {do }}$ Coautor: Antonio Erick, Wong-Díaz / ORC ID: 0000-0001-7901-5894

ID $3^{\text {er }}$ Coautor: Gabriela, Aguilar-Ortiz / ORC ID 0000-0003-3055-5712, CVU CONACYT ID: 730590

DOI: $10.35429 /$ JOIE.2019.12.3.9.16

Recibido 19 de Marzo, 2019; Aceptado 30 de Junio, 2019

\section{Resumen}

En este artículo se presenta el desarrollo de un prototipo electrónico para rastreo de vehículos basado en localización GPS como una propuesta de solución al problema de robo de vehículos que aqueja al municipio de Oaxaca de Juárez y sus municipios conurbados, de acuerdo con estadísticas de la Fiscalía Especializada en Delitos de Alto Impacto, los robos de vehículos se han incrementado aceleradamente en los últimos años.

El prototipo se desarrolla utilizando el diagrama en "V" y propone una solución al alcance del bolsillo de los dueños de vehículos, ya que productos similares requieren el dispositivo y el pago de una membresía para hacer el seguimiento en tiempo real. El prototipo consta de una placa Arduino, un módulo GPS, un módulo de comunicación SIM800 para envío de mensajes SMS, un acelerómetro para detectar movimientos del vehículo y otros componentes que se requieren para el funcionamiento del circuito completo. El programa se ha desarrollado en lenguaje $\mathrm{C}$ para Arduino y se está utilizando una aplicación gratuita de GoogleMaps para enviar la localización del vehículo al momento de detectar algún movimiento del mismo.

\begin{abstract}
This article presents an electronic prototype for vehicle tracking based on GPS (Global Positioning System) location as a proposed solution to the problem of vehicle theft that affects the Oaxaca de Juarez municipality and its surrounding municipalities, according to statistics from the Office of the Prosecutor Specialized in High Impact Crimes, vehicle thefts have increased rapidly in recent years. The "V" diagram was used to make the prototype, which proposes a solution within reach of the owners of vehicles, since similar products require the device and the payment of a membership to follow up in real time. The prototype consists of an Arduino board, a GPS module, a communication module for sending SMS (Short Message Service) messages, an accelerometer to detect vehicle movements and other components that are required for the operation of the complete circuit. The program has been developed in $\mathrm{C}$ language for Arduino and a free application of GoogleMaps is being used to send the location of the vehicle when detecting any vehicle movement
\end{abstract}

Anti-theft device, Arduino, GPS

Citación: MORALES-HERNÁNDEZ, Maricela, CABALLERO-JULIAN, Franco Gabriel, WONG-DÍAZ, Antonio Erick y AGUILAR-ORTIZ, Gabriela. Prototipo electrónico simple y de bajo costo para rastreo de vehículos basado en GPS. Revista de Ingeniería Innovativa. 2019. 3-12: 9-16

\footnotetext{
*Correspondencia al Autor (Correo electrónico: moralesh.maricela @ gmail.com)

$\dagger$ Investigador contribuyendo como primer Autor.
} 


\section{Introducción}

El presente documento describe el desarrollo de un prototipo para rastreo de vehículos basado en GPS - Global Positioning System (Hofmann, Lichtenegger y Collins, 2012), la intención es construir, a partir de componentes de bajo costo, o bien, componentes reciclados, un prototipo funcional que sea capaz de rastrear un vehículo cuando este ha sido movido del lugar donde el propietario lo haya dejado.

Este trabajo tiene como objetivo coadyuvar en la prevención de robo de vehículos que, de acuerdo con las autoridades policiacas, en el estado de Oaxaca es un problema que se ha ido incrementando de manera alarmante en los últimos años.

Cuando el prototipo desarrollado detecta un movimiento inusual en el vehículo, envía un mensaje SMS - Short Message Service (Fue y Tarimo, 2012), a un número de teléfono celular, el cual ha sido registrado previamente para poder recibir las notificaciones; en este mismo mensaje, se agrega una liga que abre directamente la aplicación de mapas de Google y se obtiene la ubicación específica en tiempo real del vehículo.

El prototipo que se propone está basado en un microcontrolador montado en una placa Arduino. El microcontrolador no es más que un circuito integrado en cuyo chip interno están implementados todos los módulos que componen la unidad central de proceso (Angulo, Angulo y Prieto, p.206). En este caso se está usando una arquitectura de código abierto como lo es Arduino, para la primera fase de la investigación; sin embargo, en un futuro, se tiene planeado sustituir esta placa por un diseño propio utilizando directamente un microcontrolador que permita un tamaño más pequeño del prototipo. Y también, se pueda tener una arquitectura y marca propia, con lo que se podría disminuir el costo del prototipo.

Este primer prototipo es el resultado de la primera fase de investigación y experimentación con circuitos programables que apoya la formación de ingenieros en electrónica y en sistemas computacionales del Instituto Tecnológico de Oaxaca; al ser una investigación que continúa en desarrollo no se ha registrado con derecho de autor.
En una siguiente fase, como se explicó en párrafos anteriores, se trabajará un diseño propio, el cual podrá registrarse con derechos de autor. La presente investigación contribuye a la búsqueda de soluciones alternativas a problemas reales, cuyo costo puede ser menor a las alternativas que ofrecen los dispositivos comerciales actuales, y de acuerdo con investigaciones desarrolladas en diferentes países como por ejemplo Sinisterra (2018) en Colombia, quien desarrolló un estudio donde demuestra la factibilidad financiera de un circuito electrónico de seguridad antirrobo en los vehículos de gasolina y diésel; proporciona las bases para que de manera análoga se pueda aplicar un estudio similar a la presente propuesta cuando se hayan concluido todas las fases de la investigación y se tenga un prototipo final.

Para el diseño de un sistema embebido o sistema programable, según Bailey (2005), se debe tener presente primero la implementación del hardware, como el diseño de un prototipo. Este prototipo debe tener un sistema de control (microcontrolador), y un conjunto de elementos de hardware que desarrollen las funciones requeridas, además debe desarrollarse también el software para programar el microcontrolador y finalmente se debe contar con una fuente de alimentación adecuada para el sistema embebido.

Para explicar el desarrollo que se ha realizado del prototipo electrónico para vehículos basado en GPS, el presente artículo se ha dividido en varias secciones, en la primera se da una breve introducción al lector para que conozca a grandes rasgos el artículo; a continuación, se plantea el problema que se está abordando; posteriormente se plantea una solución que se pretende pueda ser una alternativa para el usuario de vehículos que están en riesgo; en seguida, se explica la metodología utilizada que para el caso de sistemas embebidos o programables la literatura sugiere el "Diagrama en V" (Oshana y Kraeling, 2013).

Más adelante se explica cómo se fue desarrollando el prototipo, basándose en el diagrama en "V", para después presentar los resultados obtenidos hasta el momento. 
En las secciones finales se agradece a la Institución que nos ha dado las facilidades para desarrollar el prototipo y continuar el trabajo involucrando a los estudiantes en los proyectos de investigación; también se presentan las conclusiones a las que se llegan después de haber realizado este primer prototipo. Resaltando los trabajos futuros por desarrollar y finalmente se presentan las referencias utilizadas en el documento.

\section{Planteamiento del problema}

En el municipio de Oaxaca de Juárez y sus municipios conurbados, como en muchas otras regiones de nuestro país, existe un problema de gran impacto social, el robo de vehículos. De acuerdo con Santiago (2017) en su artículo "Van mil 500 autos robados en Oaxaca; superan cifra de 2016", las estadísticas de enero a octubre de 2017 muestran 1500 automóviles robados, 500 más que en el año 2016.

Por lo que éste problema representa una gran preocupación para todos los niveles de gobierno: municipal, estatal y federal. Ya que a partir de este delito se desencadenan otros más en donde los vehículos robados se ven involucrados. A partir de esta problemática se propone una alternativa de solución, aplicando sistemas embebidos.

\section{Propuesta de solución}

Es importante que la sociedad civil tome medidas preventivas para combatir el delito de robo de vehículos, por lo que en el presente trabajo se propone una solución utilizando sistemas embebidos.

La idea es proporcionar un sistema electrónico simple y de bajo costo basado en GPS que sea capaz de rastrear los vehículos mientras el usuario se encuentra alejado de los mismos, de tal manera, que cuando el sistema detecte un movimiento inusual, éste envíe un mensaje SMS a un número de teléfono móvil registrado en el sistema. En el mensaje se recibe la localización geográfica del vehículo: altitud y longitud; y con dicha información, utilizando el sistema de posicionamiento global, se pueda localizar el vehículo en tiempo real en GoogleMaps.
El propietario del vehículo puede entonces darle seguimiento al mismo, ya que es configurable el tiempo entre mensaje y mensaje. De este modo, se facilita la ubicación del vehículo y la ruta que está siguiendo.

\section{Metodología a desarrollar}

Para el desarrollo del presente trabajo se utiliza la metodología en "V" o diagrama en "V" como es llamado por diferentes autores. En la Figura 1, se representa el ciclo de vida que sigue un sistema para su desarrollo; es importante destacar que la progresión en el tiempo se presenta de izquierda a derecha, y que este ciclo es iterativo, se realiza tantas veces como sea necesario hasta obtener un producto adecuado y acorde con los requisitos del usuario (Oshana y Kraeling, 2013).

Este diagrama es una técnica de desarrollo rápido, y es deseable que se reduzca al mínimo el número de iteraciones para un diseño requerido (Oshana y Kraeling, 2013).

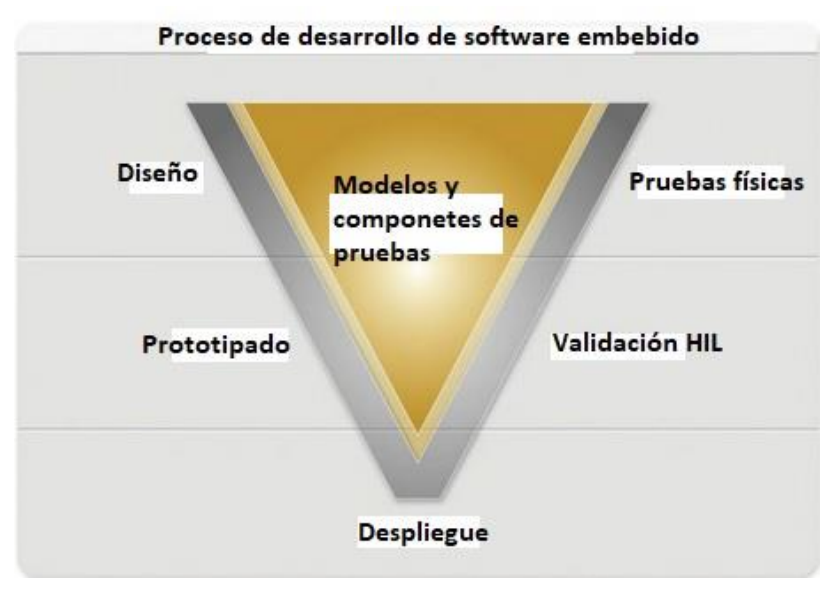

Figura 1 Diagrama en "V"

Fuente: Oshana y Kraeling, 2013

Se ha aplicado esta metodología, ya que de acuerdo con Oshana y Kraeling, ésta se adapta a las características de los sistemas embebidos, donde se requiere soluciones rápidas $\mathrm{y}$ concretas.

\section{Desarrollo}

Como se menciona en la sección previa, se utiliza el diagrama en "V" en el desarrollo del presente trabajo, y aunque se siguieron los pasos principales, en algunos momentos se adaptó de acuerdo con las necesidades del trabajo específico. En los siguientes párrafos, se encuentra esta descripción. 


\section{Investigación preliminar}

Según las estadísticas de la fiscalía especializada de delito de alto impacto, van mil quinientos autos robados en Oaxaca; cifra que supera la de 2016, las estadísticas abarcan desde enero del 2017 al mes de octubre, el titular de la dependencia, Juventino Pérez Gómez informó que en Oaxaca diariamente se registran de 5 a 10 denuncias de robo de vehículos. El servidor público dijo que la mayoría de estos ilícitos se cometen en los Valles Centrales de Oaxaca y asevera que las bandas no tienen un modus operandi definido para cometer el delito. Por este delito han sido detenidas 50 personas, los infractores son tanto de Oaxaca como otros estados (Santiago, 2017).

El 2018 está en aumento, y ya se registra en Oaxaca más de dos mil robos de autos y motocicletas. En 2017, menos del 50\% de unidades robadas fueron recuperadas. Según registros de las autoridades de seguridad pública y procuración de justicia. La zona donde más se registran el robo de unidades, al menos en $10 \%$ más que otros puntos de la entidad, es en Santa Cruz Xoxocotlán, Oaxaca de Juárez y Santa Lucía del Camino.

La última estadística de la Agencia Estatal de Investigaciones (AEI) mostró que tan sólo en el 2017, se registró un total de mil 412 autos robados y al menos mil 149 recuperados. Por mes, el organismo registró la denuncia de 116 automóviles robados en enero, 141 en febrero, 119 en marzo, 138 en abril, 118 en mayo, 134 en junio, 122 en julio, 136 en agosto, 103 en septiembre, 85 en octubre, 81 noviembre y 119 en diciembre (Sosa, 2018).

Por otro lado, se revisó lo que se ofrece comercialmente y existe una gama de productos que son semejantes al que nos ocupa en el presente trabajo; por ejemplo: ANDE Rastreador de GPS Dispositivo Anti-ladrón de rastreo de GPS Localizador de SMS Seguimiento Global en Tiempo Real para automóvil, HUAXING Rastreador de GPS, GSM/GPRS/GPS, ASG Rastreador de automóviles GPS Control Remoto, XHYA Rastreador de conducción (Amazon, 2019), entre otros. Los precios de estos oscilan entre los $\$ 1,000$ y $\$ 2,500$ pesos mexicanos.
La intención de desarrollar una propuesta alternativa es mejorar el precio, buscando componentes de bajo costo o reciclados y ampliar sus funcionalidades; utilizando para todo ello, arquitecturas y software de código abierto.

\section{Diseño del prototipo}

El prototipo se diseña con las características necesarias que le permitan recuperar la ubicación exacta de cualquier vehículo y enviar la posición al propietario, a través de un SMS. Para tal fin, el prototipo incluye un módem GPS que obtiene dicha ubicación en términos de longitud y latitud. Estos datos se envían a la placa Arduino, la cual está conectada con un módem GSM., el cual envía los mensajes usando el protocolo de comunicación SMS al número de teléfono móvil que ha sido registrado previamente en el sistema, en intervalos periódicos establecidos en la programación del prototipo. También se requiere de un teléfono móvil con tecnología 3G para establecer la comunicación usando el protocolo de comunicación SMS como se menciona anteriormente. En la figura 2 se presenta el diagrama a bloques del diseño del prototipo, incluyendo los elementos que se han descrito en los párrafos anteriores.

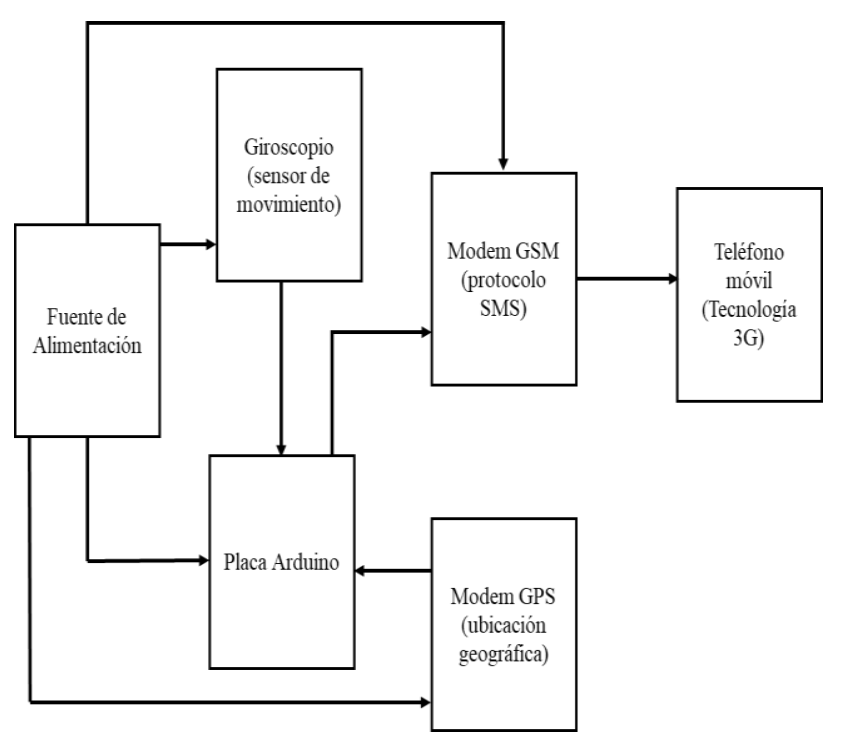

Figura 2 Diagrama a bloques del prototipo Fuente: Elaboración Propia

La fuente de alimentación mostrada en la figura 2 es de 5 volts por 1 ampere. Cabe hacer hincapié que para que el módem GSM tenga cobertura para enviar mensajes se requiere de una tarjeta SIM con pago de servicios al proveedor de telefonía móvil. 
Así mismo puede verse en la misma figura que se requiere un teléfono móvil con tecnología $3 \mathrm{G}$ o $4 \mathrm{G}$, que es la tecnología con la cual se intercambian los mensajes SMS.

\section{Simulación del prototipo}

Antes de conectar físicamente los componentes, se realiza una simulación del prototipo, para ello se utiliza el simulador en línea tinkercad, éste se localiza en http:// www.tinkercad.com/. En la figura 3 se muestra el prototipo simulado; sin embargo, antes de poder probarlo se requiere programar la placa Arduino.

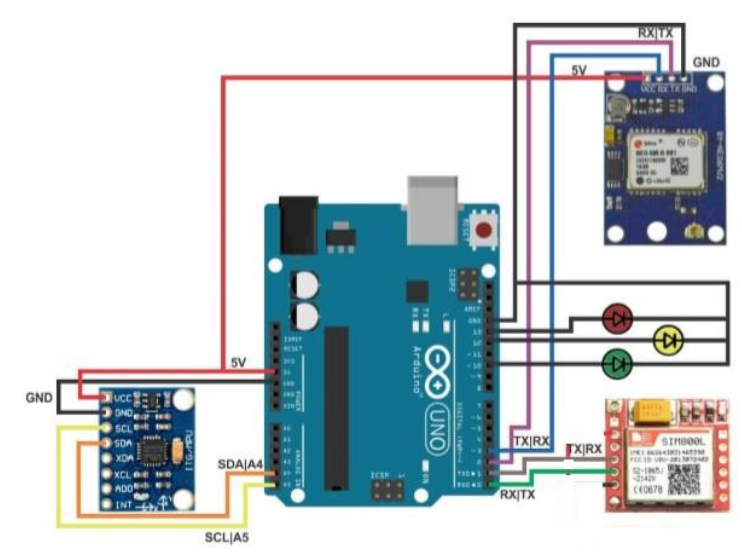

Figura 3 Simulación del prototipo

Fuente: Elaboración Propia

En la tabla 1 se muestra un fragmento de la programación de la placa Arduino, éste corresponde a las librerías para el manejo del giroscopio y el módulo SIM de comunicación.

\#include <I2Cdev.h>// Librerias I2C para controlar el mpu6050

\#include <MPU6050.h>// la libreria MPU6050.h necesita I2Cdev.h, I2Cdev.h necesita Wire.h

\#include <Wire.h>

\#include $<$ SoftwareSerial.h $>/ /$ Libreria para establecer una segunda conexion serial

\#include < TinyGPS.h>//Libreria para controlar y obtener datos del GPS

// La dirección del MPU6050 puede ser 0x68 o 0x69, dependiendo

// del estado de AD0. Si no se especifica, 0x68 estará implicito

MPU6050 sensorMPU;

TinyGPS gps;

SoftwareSerial SIM800L(2,3);//Se establecen los pines D4=RX y D5=TX - Comunicación serial con el módulo SIM800L

//Declaracion de variables

bool newData = false;

unsigned long chars;

unsigned short sentences, failed;

float flat,flon;
// Valores RAW (sin procesar) del acelerometro y giroscopio en los ejes $\mathrm{x}, \mathrm{y}, \mathrm{z}$

int ax, ay, az;

int gx, gy, gz;

//Declaracion del mensaje a enviar mediante el SIM800L String msj; String notify;

Tabla 1 Fragmento de código de Arduino

Fuente: Naylamp Mechatronics, 2016

\section{Construcción del prototipo físico}

Una vez que se realizan las pruebas de simulación del prototipo, se genera la lista de componentes de hardware, los cuales se presentan en la tabla 2, también se presentan los precios aproximados de los componentes, pero cabe mencionar, que éstos pueden variar con el tiempo.

\begin{tabular}{|l|l|r|}
\hline Componente & Fabricante & \multicolumn{1}{l|}{ Costo } \\
\hline Módulo SIM800L & $\begin{array}{l}\text { SIMCom Wireless } \\
\text { Solutions Co.,Ltd. }\end{array}$ & $\$ 173.00$ \\
\hline Módulo GPS Neo-6M & u-blox Company. & $\$ 189.00$ \\
\hline $\begin{array}{l}\text { Acelerómetro } \\
\text { mpu6050 }\end{array}$ & $\begin{array}{l}\text { InvenSense, a TDK } \\
\text { Group Company. }\end{array}$ & $\$ 55.00$ \\
\hline $\begin{array}{l}\text { Cristal oscilador } \\
\text { 8Mhz }\end{array}$ & IQD Frequency & $\$ 8.00$ \\
\hline $\begin{array}{l}\text { Cables con conectores } \\
\text { macho y hembra }\end{array}$ & & $\$ 15.00$ \\
\hline Placa Arduino Uno & Arduino & $\$ 160.00$ \\
\hline Protoboard & Steren & $\$ 140.00$ \\
\hline
\end{tabular}

Tabla 2 Lista de componentes

Fuente: Elaboración Propia

Ya que se tienen los componentes disponibles se inicia el armado del prototipo en tarjeta de prueba, éste se muestra en la figura 4.

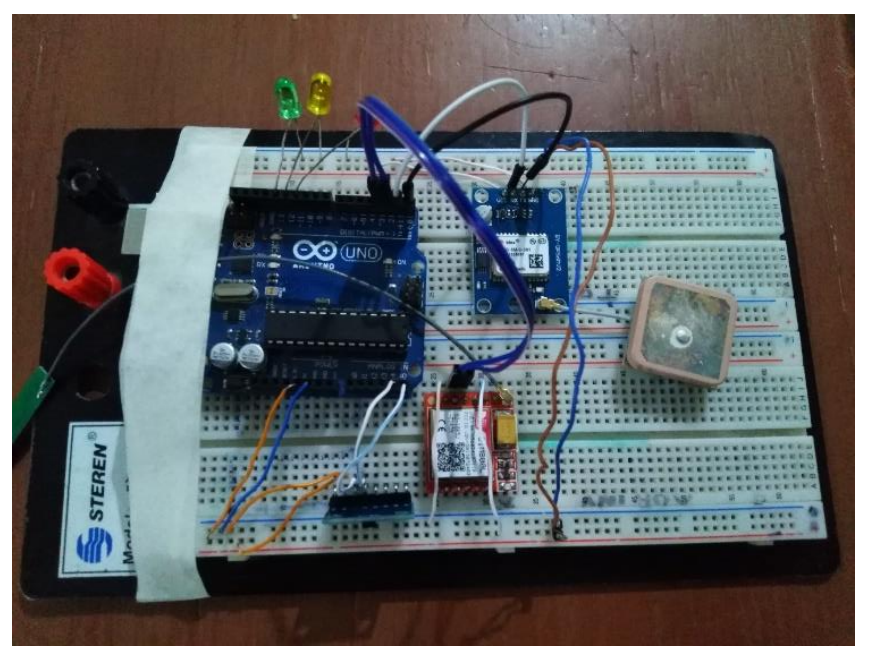

Figura 4 Prototipo físico armado Fuente: Elaboración Propia 
Con el prototipo armado en la tarjeta de prueba, se carga el programa a la placa Arduino, el cuál previamente se desarrolla, en éste se configura cada componente y posteriormente se programa la lectura del sensor (giroscopio) y se envía la señal que requiere el Módem GPS y el Módem GMS para poder generar los mensajes SMS que deben llegar al dispositivo móvil o celular con la información de ubicación del automóvil en coordenadas de altitud y longitud que se interpretan en el mapa de Google.

\section{Pruebas físicas realizadas al prototipo}

Al prototipo se le aplican varias pruebas: (1) prueba de continuidad, (2) prueba de integrado con multímetro, (3) prueba de voltaje y (4) prueba de integración. En los siguientes párrafos se describe cada una de ellas.

1. Prueba de continuidad. En electrónica, una prueba de continuidad es la verificación de un circuito eléctrico para ver si fluye la corriente. Se realiza una prueba de continuidad colocando una pequeña tensión (conectada en serie con un LED o componente que produce ruido, como un altavoz piezoeléctrico), a través de la ruta elegida. Si el flujo de electrones es inhibido por conductores rotos, componentes dañados o resistencia excesiva, el circuito está "abierto". Se aplica la prueba al prototipo y se obtiene un resultado positivo, lo cual indica que éste pasa la prueba de continuidad.

2. Prueba de integrado. Esta prueba consiste en revisar que la placa Arduino no tenga algún daño físico aparente, posteriormente se verifica que la alimentación se encuentra en los niveles correctos de acuerdo con la hoja de datos proporcionada por el fabricante. A continuación, se requiere verificar que las señales de entrada/salida estén correctas, concentrando las mediciones en las señales que se van a utilizar en el prototipo. En el caso del presente prototipo se tuvieron algunos problemas con los componentes periféricos como el módulo de comunicación GSM, que en dos ocasiones sufrió daños y no operó con normalidad. La solución que se le dio para superar la prueba de integrado fue sustituirlo por otro módulo.
3. Prueba de voltaje. Esta prueba se realiza para verificar si la tensión en diferentes terminales está de acuerdo con el requisito o no, en el caso de la placa Arduino, ésta ya contiene las salidas de 5 y 3 volts para alimentar circuitos, tomando la corriente a través del equipo de cómputo con una conexión USB. Por lo que ésta prueba concluye con éxito.

4. Prueba de integración. Finalmente se aplica esta prueba, que en este caso, se cargó el programa completo en la placa Arduino, se alimentó correctamente todo el prototipo y se inicia su funcionamiento con todos los componentes, desde luego no tuvo el éxito esperado en la primera prueba, pero después de arreglar detalles que se presentaban, se logró pasar la prueba y todo funciona como se planteó. Por lo que, esta prueba también fue superada.

Con las pruebas aplicadas al prototipo se concluyó la etapa de desarrollo y en la siguiente sección se explican los resultados obtenidos.

\section{Resultados}

Al finalizar la construcción y prueba del prototipo, se logra lo siguientes:

1. Un prototipo funcional que, al detectar un movimiento inusual en el mismo, el cual es sensado por el giroscopio; permite enviar señales que habilitan el modem GPS para leer la ubicación en tiempo real que se tiene.

2.

El prototipo, entonces, envía una señal al modem GSM para que, estos datos sean colocados en un mensaje de texto SMS, éste mensaje es recibido en el número de dispositivo móvil que se configura en la programación de la placa Arduino. En este punto también se prueban diferentes números con el fin de corroborar el correcto funcionamiento del prototipo. 
3. El módem GSM en conjunto con la tarjeta SIM envía un mensaje al dispositivo móvil, como se muestra en la figura 5. En la figura se puede notar que se recibe un mensaje que conecta los datos directamente al mapa de Google con el fin de que el usuario obtenga una ubicación inmediata y directa en un mapa y pueda darle seguimiento a la unidad en cuestión. Como puede observarse en la figura 5, se simuló el movimiento del prototipo a diferentes puntos dentro de un radio no mayor a 100 metros y la ubicación recibida varía de acuerdo a la posición física del prototipo.

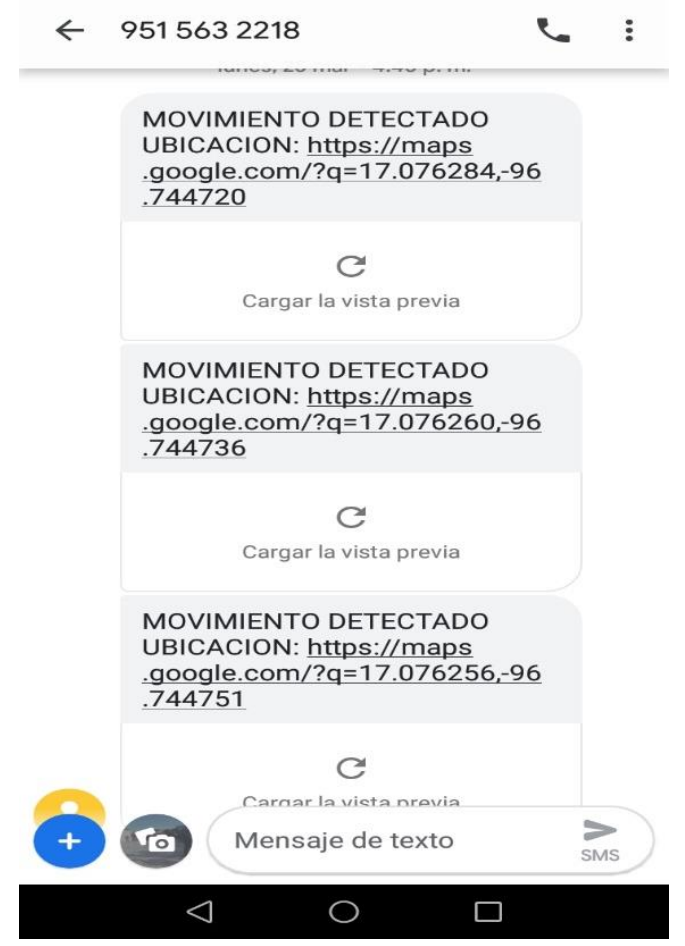

Figura 5 Mensaje SMS recibido en el teléfono celular Fuente: Elaboración Propia

4. Finalmente, como resultado de la lectura de ubicación geográfica, el prototipo dentro del mensaje SMS, agrega una liga para ir a la ubicación en el mapa de Google, tal como se muestra en la figura 6 , donde puede verse la altitud y longitud que el prototipo lee del GPS, al momento de detectar un movimiento en el vehículo al que se le da el seguimiento.

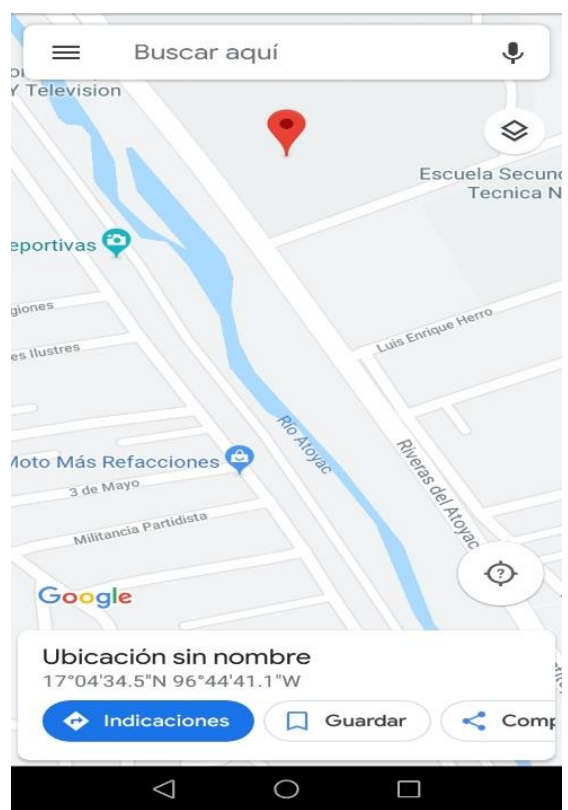

Figura 6 Ubicación geográfica en Google Maps Fuente: Elaboración Propia

\section{Agradecimientos}

En el desarrollo del presente trabajo se agradece al Tecnológico Nacional de México, que a través del Instituto Tecnológico proporcionó el apoyo y las facilidades para el desarrollo de la investigación y elaboración del presente artículo; así como el apoyo para su divulgación, otorgando el crédito correspondiente a las instituciones. También se agradece ampliamente la colaboración y dedicación de los autores del artículo, profesores y estudiantes que participan en la investigación.

\section{Conclusiones}

Al realizar este primer prototipo para un dispositivo antirrobo se lograron los objetivos planteados originalmente, la idea de tener una tecnología que pueda patentarse y no usar los dispositivos comerciales, le da una ventaja al desarrollo de esta investigación, ya que se pretende mejorar este primer prototipo, de tal modo que se vaya desarrollando tecnología propia; la cual, pueda tener una marca propia.

En este momento el costo del prototipo es elevado, pero se plantea la posibilidad incluso de reutilizar algunos componentes de dispositivos que aparentemente han caído en la obsolescencia, pero que con los mensajes SMS, no se requiere que los dispositivos móviles sean de última tecnología para poder recibirlos. 
Esto ofrece una gran ventaja sobre otros dispositivos que requieren de tecnologías de última generación, en su lugar, se usa la tecnología de comunicación básica para que los mensajes puedan intercambiarse sin ningún problema.

Por otro lado, se sabe que, en el estado de Oaxaca, por las estadísticas consultadas, ocurren diariamente robo de vehículos y el presente prototipo puede coadyuvar en el abatimiento de este delito.

\section{Trabajos futuros}

Una siguiente etapa que se ha pensado es cambiar la placa Arduino por un arreglo que incluya un microcontrolador de bajo costo y con características mínimas que habiliten las funcionalidades deseadas en el prototipo. Hacer un rediseño y tener un circuito propio que pueda ser registrado con derechos de autor.

Por otro lado, también se plantea que el dispositivo pueda ser autorecargable, que éste se pueda recargar cuando el automóvil esté en funcionamiento y cuando deje de moverse, tendrá una recarga de larga duración. Esta funcionalidad requiere de una investigación amplia sobre pilas y fuentes de energía autónomas que garanticen una duración adecuada para el uso que se le dará al dispositivo de rastreo.

Otro de los trabajos futuros a desarrollar es que el prototipo pueda interrumpir el funcionamiento del motor en caso de que éste haya sido robado. Esta funcionalidad tendrá que ver con la activación de actuadores en el sistema embebido y que pueda adaptarse a los modelos de vehículos que aún no cuentan con esta tecnología desde su fabricación.

Por otro lado, también se tiene la posibilidad de extender la funcionalidad a casi cualquier vehículo o bien que el dispositivo se adapte a diferentes tipos de vehículos

\section{Referencias}

Amazon (2019). Departamento de electrónicos: Localizadores GPS. Recuperado de https://www.amazon.com.mx/
Angulo, J.M., Angulo, I. y Prieto, M.A. (2010). Electrónica Digital y Microprogramable. España: Paraninfo.

Autodesk Tinkercad. (2019). Circuitos: Añade luz y movimiento a tus diseños 3D con circuitos electrónicos. Recuperado de https://www.tinkercad.com/learn/circuits.

Bailey, O. (2005). Embedded Systems: Desktop Integration. USA: Wordware Publishing, Inc.

Fue, K, y Tarimo, C. (2012). Design and implementation of the remote control system using SMS via GSM for home electrical system. Reino Unido: Lulu.com.

Hofmann, B., Lichtenegger, H. y Collins, J. (2012). Global Positioning System: Theory and Practice. USA: Springer Science \& Business Media.

Naylamp Mechatronics. (16 de abril de 2016). Tutorial MPU6050, Acelerómetro y Giroscopio. Recuperado de https://naylampmechatronics.com/blog/45_Tuto rial-MPU6050-Aceler\%C3\%B3metro-yGiroscopio.html

Oshana, R. y Kraeling, M. (2013). Software Engineering for Embedded Systems: Methods, Practical Techniques, and Applications. USA: Elsevier, Inc.

Santiago, J. (23 de noviembre de 2017). Van mil 500 autos robados en Oaxaca; superan cifra de 2016. NVI Noticias Oaxaca. Recuperado de https://www.nvinoticias.com/nota/77117/vanmil-500-autos-robados-en-oaxaca-supera-cifrade-2016

Sinisterra, H. (2018). Estudio de factibilidad para el desarrollo de un circuito electrónico de seguridad antirrobo en los vehículos de gasolina y diésel. Colombia: Fundación Universitaria Católica Lumen Gentium. Facultad de Ciencias Empresariales.

Sosa, Y. (27 de enero de 2018). Registra Oaxaca más de 2 mil robos de autos y motocicletas. El imparcial de Oaxaca. Recuperado de http://imparcialoaxaca.mx/oaxaca/116768/registraoaxaca-mas-de-2-mil-robos-de-autos-ymotocicletas/ 